\title{
Stellar population models in the UV
}

\section{Characterisation of the New Generation Stellar Library ${ }^{\star}$}

\author{
M. Koleva ${ }^{1,2,3,4, \star \star}$ and A. Vazdekis ${ }^{1,2}$ \\ 1 Instituto de Astrofísica de Canarias, La Laguna, 38200 Tenerife, Spain \\ e-mail: mina.koleva@gmail.com \\ 2 Departamento de Astrofísica, Universidad de La Laguna, 38205 La Laguna, Tenerife, Spain \\ 3 Université Lyon 1, Villeurbanne, 69622, France; CRAL, Observatoire de Lyon, 69561, St. Genis Laval, CNRS, UMR 5574, France \\ 4 Sterrenkundig Observatorium, Ghent University, Krijgslaan 281, S9, 9000 Ghent, Belgium
}

Received 10 September 2011 / Accepted 27 October 2011

\section{ABSTRACT}

\begin{abstract}
Context. The spectral predictions of stellar population models are not as accurate in the ultra-violet (UV) as in the optical wavelength domain. One of the reasons is the lack of high-quality stellar libraries. The New Generation Stellar Library (NGSL), recently released, represents a significant step towards the improvement of this situation.

Aims. To prepare NGSL for population synthesis, we determined the atmospheric parameters of its stars, we assessed the precision of the wavelength calibration and characterised its intrinsic resolution. We also measured the Galactic extinction for each of the NGSL stars.

Methods. For our analyses we used ULySS, a full spectrum fitting package, fitting the NGSL spectra against the MILES interpolator. Results. We find that the wavelength calibration is precise up to $0.1 \mathrm{px}$, after correcting a systematic effect in the optical range. The spectral resolution varies from $3 \AA$ in the UV to $10 \AA$ in the near-infrared (NIR), corresponding to a roughly constant reciprocal resolution $R=\lambda / \delta \lambda \approx 1000$ and an instrumental velocity dispersion $\sigma_{\text {ins }} \approx 130 \mathrm{~km} \mathrm{~s}^{-1}$. We derived the atmospheric parameters homogeneously. The precision for the FGK stars is $42 \mathrm{~K}, 0.24$ and 0.09 dex for $T_{\text {eff }}, \log g$ and $[\mathrm{Fe} / \mathrm{H}]$, respectively. The corresponding mean errors are $29 \mathrm{~K}, 0.50$ and 0.48 dex for the $\mathrm{M}$ stars, and for the OBA stars they are 4.5 percent, 0.44 and 0.18 dex. The comparison with the literature shows that our results are not biased.
\end{abstract}

Key words. methods: data analysis - stars: fundamental parameters

\section{Introduction}

The stellar libraries are collections of spectra that share identical spectral coverage and resolution. They have several important applications: they are used as references to classify stars and to determine their atmospheric parameters (e.g. Wu et al. $2011 \mathrm{~b}$, and the reference therein), as templates to recover the line-of-sight velocity distribution of galaxies (e.g. Cappellari \& Emsellem 2004), or to calibrate photometry (see Bessell 2005, for a review). They are one of the critical ingredients in the stellar population synthesis (e.g. Vazdekis et al. 2010). To produce high-quality stellar population in the blue is the goal of this series of papers.

The stellar libraries can be theoretical or observational. The theoretical libraries can, in principle, be computed for any value of temperature, gravity, metallicity and detailed chemical composition, and the resolution is essentially limited by the computing power (e.g. Kurucz 1979; Hauschildt et al. 2003; Palacios et al. 2010). They would be the ideal references if they were able to reproduce the observations accurately. In fact, the physical approximations (1D, LTE, convection,...) and the lack of complete databases of atomic and molecular transition result in discrepancies between these stellar models and

\footnotetext{
* Table A1 is only available at CDS via anonymous ftp to cdsarc.u-strasbg.fr $(130.79 .128 .5)$ or via

http://cdsarc.u-strasbg.fr/viz-bin/qcat?]/A+A/538/A143

$\star \star$ Marie Curie fellow.
}

observations (Martins \& Coelho 2007; Prugniel et al. 2011; Beifiori et al. 2011). The observational libraries, on the other side, have the advantage to be assembled from real stars, but they suffer from instrumental limitations (finite resolution) and limited atmospheric parameter coverage. Because these libraries are constructed from Galactic stars, they are bound to the chemical composition found in the Galaxy, and more specifically that of the solar neighbourhood. It is possible to combine the observed and theoretical libraries to predict the differential effect of changing some physical ingredients. These semi-empirical libraries were used to extend the range of the parameter space (Prugniel et al. 2011) or to compute the effect of a variable abundance of $\alpha$-elements in models of stellar populations (Cervantes et al. 2007; Prugniel et al. 2007a; Walcher et al. 2009).

The most important property of the stellar libraries is the coverage of the atmospheric parameters, such as effective temperature, gravity, metallicity. Recently, the detailed abundances are beginning to be considered as important parameters, too. Other properties to be considered are the spectral resolution, the wavelength coverage, the flux, and the wavelength calibration of the spectra. Fortunately, we have optical libraries that cover the parameter space reasonably well: ELODIE (Prugniel \& Soubiran 2001; Prugniel et al. 2007b), CFLIB (Valdes et al. 2004), and MILES (Sánchez-Blázquez et al. 2006). These empirical libraries have a fair spectral resolution $(\lambda / \Delta \lambda=R \sim$ 2000-10 000) that is compatible with the resolution of the most widely used optical spectrographs for galactic studies, and they 
have a good flux calibration (except CFLIB, Bruzual A. 2007). They are built from normal stars in all luminosity classes and spectral types from $\mathrm{O}$ to $\mathrm{M}$. They cover a wide range in metallicities $(-3.0 \lesssim[\mathrm{Fe} / \mathrm{H}] \lesssim 1.0)$. The stars in these three libraries, as in any other empirical library, have the abundance pattern of the solar neighbourhood (see Wheeler et al. 1989, for a review).

None of these three libraries extend further blue-ward than $3500 \AA$. The importance of the UV as a gate to understand the physics of the stellar systems was recognised back in the 1980s (e.g. Faber 1983). In particular, the UV is irreplaceable to characterise the metallicity and the star-formation history (SFH) of young populations, to study the enhancement of $\alpha$-elements or the contribution of blue horizontal branch stars to the integrated fluxes. It is also of prime importance studying distant galaxies whose restframe UV is observed in the optical, where the current instrumentation is most developed.

In a simple stellar population (SSP) the blue wavelengths are predominantly sensitive to the hottest stars. At any age greater than $10 \mathrm{Myr}$, those are the dwarfs at the main-sequence turn-off. After $\sim 1$ Gyr of evolution, the He-burning stars may become bluer (hotter) than the RR Lyrae pulsating stars and populate the so-called blue-horizontal branch (BHB). Together with the blue stragglers (BS, low-mass, main-sequence stars with excessive blue colours) they may have an important contribution to the integrated spectra (Lee et al. 2002; Cenarro et al. 2008) and mimic young populations (e.g. Maraston \& Thomas 2000; Koleva et al. 2008; Ocvirk 2010; Percival \& Salaris 2011). Our ability to distinguish between the real young stars and these exotic populations relies on their different contribution to the different parts of the spectral energy distribution (SED). Thus, combining optical and UV data can lift the degeneracy (Rose 1984; Schiavon et al. 2004; Percival \& Salaris 2011).

Blue-horizontal branch stars and blue stragglers are frequently observed in Galactic clusters and were also detected in Local Group galaxies (e.g. Mapelli et al. 2007). The presence of the BHB and BS is connected with some properties of the populations (e.g. the metallicity for the BHB) but may also be related with the environment and with some large scale properties of the host systems. Therefore, the ability to distinguish these stars in integrated spectra would be a major step toward the understanding for the genesis and evolution of stellar systems.

It has been shown that the effects of $\alpha$-elements enhanced partition are emphasised blue-ward, both in stars (e.g. Cassisi et al. 2004) and in stellar populations (Coelho et al. 2005). Thus, the blue spectral range should provide us with more diagnostic indices to better constrain the galactic star-formation histories (Serven et al. 2011).

The first attempts to gather a UV library that covered the MK sequence were made by Wu et al. (1983) and Fanelli et al. (1992) with observations from the International Ultraviolet Explorer ${ }^{1}$ (IUE). This library has a resolution of $7 \AA$ and contains 218 stars of essentially solar metallicity. Still, this first UV library is quite limited compared to its modern optical counterparts. With the new generation stellar library (NGSL, Gregg et al. 2006) the gap between the optical and UV libraries begins to narrow.

The New Generation Spectral Library ${ }^{2}$ is a major step towards the modelling of the stellar populations in the UV. It was observed with the Hubble Space Telescope Imaging Spectrograph (STIS) and consists of 374 stars with metallicities between $-2.0 \mathrm{dex}$ and $0.5 \mathrm{dex}$. As its optical counterparts it contains normal stars from $\mathrm{O}$ to $\mathrm{M}$ spectral types in all

\footnotetext{
1 http://archive.stsci.edu/iue/

2 http://archive.stsci.edu/prepds/stisngsl/
}

luminosity classes. Its wavelength coverage from 0.2 to $1.0 \mu \mathrm{m}$ is the widest available amongst the observational libraries at this resolution, though it does not go as far in the UV as IUE and misses Ly $\alpha$. Its spectral resolution is $R \sim 1000$. The stars of the NGSL were rigorously chosen to have a good coverage in the space of atmospheric parameters. Heap \& Lindler (2010) measured the atmospheric parameters for most NGSL stars using ATLAS9 model atmospheres (Castelli \& Kurucz 2004) as templates (46 stars, i.e. 12 percent of the sample, miss one or more parameters).

In order to implement this library in population models, its characteristics have to be assessed accurately. This is the goal of the present paper. In Sect. 2 we present the data, while in Sect. 3 we present our methodology. In Sect. 4 we characterise the linespread function (LSF) of the NGSL. In Sect. 5 we derive the atmospheric parameters homogeneously and compare them with the literature. In Sect. 6 we measure the spectroscopic Galactic extinction of the stars. Finally, our conclusions and prospects are presented in Sect. 7.

\section{The NGSL spectra}

The NGSL stars were observed with STIS on-board HST with three different gratings (G230LB, G430L and G750L),

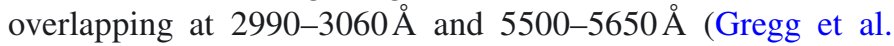
2006). The final spectra cover the wavelength range from $\sim 0.2$ to $\sim 1.0 \mu \mathrm{m}$ (slightly different from star to star) and have a resolution of $R \sim 1000$. The flux-calibration reaches a precision of 3 percent (Heap \& Lindler 2009). The spectra are calibrated in air wavelengths, with sampling varying as follows: $1.373 \AA / \mathrm{px}\left(\lambda \lambda 1675-3060 \AA\right.$ or $165 \mathrm{~km} \mathrm{~s}^{-1}$ at $2500 \AA), 2.744 \AA / p x\left(\lambda \lambda 3060-5650 \AA\right.$ or $205 \mathrm{~km} \mathrm{~s}^{-1}$ at $\left.4000 \AA\right)$, $4.878 \AA / \mathrm{px}\left(\lambda \lambda 5650-10196 \AA\right.$ or $183 \mathrm{~km} \mathrm{~s}^{-1}$ at $\left.8000 \AA\right)$. Details about the data reduction can be found in http://archive. stsci . edu/pub/hlsp/stisngsl/aaareadme.pdf. We downloaded version 2 of the reduced data.

The stars were chosen to sample four metallicity groups, roughly 150 stars in each bin: $[\mathrm{Fe} / \mathrm{H}]<-1.5 ;-1.5<[\mathrm{Fe} / \mathrm{H}]<$ $-0.5 ;-0.3<[\mathrm{Fe} / \mathrm{H}]<+0.1 ;+0.2<[\mathrm{Fe} / \mathrm{H}]$. The targeted sample included 600 stars. Unfortunately, about 200 stars were not observed owing to the failure of STIS in 2004. The released library lacks some hot- and low-metallicity stars, but is wellsuited to model intermediate- and old-aged stellar populations.

\section{Methodology}

We applied a full spectrum fitting approach to characterise the NGSL spectra and to infer the stellar parameters. For this purpose we employed the ULySS package (Koleva et al. 2009). We followed the approach used in Wu et al. (2011b), Prugniel et al. (2011), and Wu et al. (2011a) to derive (i) the LSF to describe the intrinsic resolution and its variation with wavelength; (ii) the atmospheric parameters of the stars; and (iii) the Galactic extinction on the line-of-sight of each star.

\subsection{Spectral fitting}

ULySS performs a parametric minimisation of the squared differences between an observation and a linear combination of nonlinear models as

$\operatorname{Obs}(\lambda)=P_{n}(\lambda) \times\left(G\left(v_{\text {sys }}, \sigma\right) \otimes \sum_{i=0}^{i=k} W_{i} \operatorname{CMP}_{i}\left(a_{1}, a_{2}, \ldots, \lambda\right)\right)$, 
where $\operatorname{Obs}(\lambda)$ is the observed one-dimensional spectrum function of the wavelength $(\lambda)$, sampled in $\log \lambda ; P_{n}$ is a multiplicative polynomial of degree $n$; and $G\left(v_{\text {res }}, \sigma\right)$ is a Gaussian broadening function parameterised by the residual velocity $v_{\text {res }}$, and the dispersion $\sigma$ (see the discussion in Sect. 4). The $\mathrm{CMP}_{i}$ are $k$ non-linear functions of any number of parameters, figuring the physical model. Their weights $W_{i}$ can be constrained (to be positive in the present case).

\subsection{Applications}

Here we will use three different specific cases of Eq. (1). First, to determine the broadening by comparing the stars in common between NGSL and a reference library, we used a single component that consists in a template spectrum (i.e. no non-linear parameter). Equation (1) degenerates to

$\operatorname{Obs}(\lambda)=P_{n}(\lambda) \times G\left(v_{\text {sys }}, \sigma\right) \otimes S(\lambda)$,

where $S(\lambda)$ is the template spectrum.

Second, to determine the broadening with respect to a theoretical library, we used a positive linear combination of spectra taken from a grid.

$\operatorname{Obs}(\lambda)=P_{n}(\lambda) \times\left(G\left(v_{\mathrm{sys}}, \sigma\right) \otimes \sum_{i=0}^{i=k} W_{i} S_{i}(\lambda)\right)$,

where $S_{i}$ are the $k$ template spectra. The weights $W_{i}$ are bound to be positive.

Finally, we measured the atmospheric parameters of the stars using a TGM component, as

$\operatorname{Obs}(\lambda)=P_{n} \times G \otimes T G M\left(T_{\text {eff }}, \log g,[\mathrm{Fe} / \mathrm{H}], \lambda\right)$,

where TGM is a model spectrum, function of the effective temperature, surface gravity and metallicity, respectively, written as $T_{\text {eff }}, \log g$, and $[\mathrm{Fe} / \mathrm{H}]$. The free parameters in the minimisation are the degree of the polynomial, $v_{\text {res }}, \sigma, T_{\text {eff }}, \log g$, and $[\mathrm{Fe} / \mathrm{H}]$.

The model used for the TGM component was the MILES interpolator, presented in Prugniel et al. (2011). This interpolator returns a spectrum for any temperature, metallicity, and gravity where each wavelength bin is computed by an interpolation over the entire reference library. It is constructed from three different sets of polynomials for the OBA, FGK and M type temperature ranges, and it is linearly interpolated in overlapping regions. Each of those sets of polynomials are valid for a wide range of parameters, which means that this is a global interpolation. The MILES interpolator (Prugniel et al. 2011) has the advantage to be derivable and continuous everywhere, which makes it suitable for non-linear minimisation as e.g. in ULySS.

The purpose of the polynomial is to absorb the discrepancies in the global shape of the energy distribution between the observation and the model, which can result from the extinction along the line-of-sight, or uncertainties in the flux calibration. The biases that a prior normalisation of the observations would introduce are minimised, because this continuum is fitted in the same time as the parameters of the model. If the flux calibration and shape of the energy distribution of the model can be trusted, $P_{n}$ can be used to estimate the extinction (Sect. 6). The choice of the polynomial degree is governed by the wavelength range, the precision of the flux calibration, the spectral resolution, and the complexity of the fitted model. Wu et al. (2011b) have shown that a degree as high as $n=100$ did not bias their determination of the atmospheric parameters of CFLIB. However, in the present case, where the spectral resolution is lower, we found that $n>25$ may affect the determination of the atmospheric parameters (the polynomial competes with the model to fit the broadest features). We investigated the dependence of the fitted atmospheric parameters with the degree of the polynomial using 10 stars from each luminosity group (OBA, FGK and M). We found that at $n=12$ the resulting atmospheric parameters are stable.

\section{Line-spread function}

In Eqs. (1) to (4) the models are convolved with $G$ to match the observation. This convolution is usually meant to account for the physical broadening of the spectrum when both the observation and the model have the same resolution. This is used to measure the internal kinematics (velocity and velocity dispersion) of galaxies (Tonry \& Davis 1979; Cappellari \& Emsellem 2004) and requires the logarithmic sampling of the spectrum. The same approach may be used to measure the line-of-sight velocity and the rotational broadening of stars.

When the two spectra have different resolutions, $G$ encompasses the physical broadening and the relative broadening between the observation and the model. This can be written as $G=G_{\text {phy }} \otimes L S F_{\text {rel }}$, where $G_{\text {phy }}$ is the physical broadening and $L S F_{\text {rel }}$ the relative LSF of the observation with respect to the template (note that the resolution of the models should be higher than that of the observation). The LSF (or relative LSF) generally depends on the wavelength, and the match of resolution cannot be written as a convolution. However, because it changes slowly with the wavelength, we can assume that Eq. (1) holds in small wavelength intervals, and the ULySS analysis of the spectrum in a series of consecutive wavelength segments will allow us to monitor the wavelength dependence of $G$. In addition, as in the present case, $G_{\text {phy }}$ is generally negligible compared to $L S F_{\text {rel }}$, this approach will allow us to derive the wavelengthdependent LSF. ( $G_{\text {phy }}$ is not negligible only for some fast rotating stars that we will exclude when computing the LSF.) The first moment of the LSF (a velocity-shift) represents the errors in the wavelength calibration and reduction to the rest-frame velocity. The second moment (instrumental velocity dispersion) represents the resolution. These two moments are likely variable throughout the library because of slightly different observing conditions (e.g. centring of the star in the slit) and/or data reduction. The dispersion relation of NGSL was determined using the stellar lines because no calibration arc-lamp exposures were available. This process may limit the precision of the wavelength calibration, and result in systematic distortion of the wavelength scale that our analysis may reveal.

The (absolute) LSF is $L S F=L S F_{\text {rel }} \otimes L S F_{\text {ref }}$, where $L S F_{\text {ref }}$ is the LSF of the reference spectra that are known or can be measured. Because in the present work we consider Gaussian LSFs, the absolute broadening can be derived by quadratically summing the broadening of the reference spectra and the relative broadening (returned from the optimisation algorithm).

The most straightforward and robust choice is to use highresolution spectra of some NGSL stars as reference to derive the LSF. Because the spectral coverage of NGSL is wider than any other library, we will perform independent comparisons in the different wavelength domains. In the optical range, we compared the stars in common with the ELODIE and MILES libraries. To complete the LSF determination in the blue, we will use the UVBlue theoretical grid (Rodríguez-Merino et al. 2005). Finally, we used a grid of theoretical spectra from Munari et al. (2005) to construct the LSF over most of the range, except for 
the first $500 \AA$ at the blue end. The different analyses were crosschecked in their wide overlapping spectral regions.

\subsection{ELODIE and MILES libraries}

To check the wavelength calibration of the NGSL stars in the optical, we compared the stars in common between NGSL and MILES $^{3}$ (Sánchez-Blázquez et al. 2006; Falcón-Barroso et al. 2011) or ELODIE (Prugniel \& Soubiran 2001; Prugniel et al. 2007b) libraries. In that case, Eq. (2) applies. Since the same objects were observed in the two libraries, the only difference between the spectra should be the instrumental broadening. We proceeded as follow: first we fitted the "observed" against the "template" star to clean residual spikes in the spectrum. Second, we fitted a Gaussian broadening in segments of $400 \AA$ separated by $300 \AA$ (so that the consecutive segments overlap by $100 \AA$ at both ends). Third, we derived the absolute LSF by adding quadratically the LSF of the reference library. For ELODIE we took a FWHM $=0.58 \AA$ and for MILES a FWHM $=2.5 \AA$ (Prugniel et al. 2011; Falcón-Barroso et al. 2011). Finally, the results were averaged to produce a mean LSF. The individual measurement outliers, from either particular stars (imprecise reduction to the rest-frame or fast rotators) or poor fits (low $\mathrm{S} / \mathrm{N}$ ) were rejected at this step using at most five iterations of a $3-\sigma$ clipping (with the IDL function MEANCLIP from the Astrolib library $\left.^{4}\right)$. We also rejected the measurements where the broadening varied significantly between two successive segments.

The results for 127 stars in common with ELODIE are plotted on Figs. 1, 2. The results from the 137 MILES comparisons are similar and consistent, though with a wider (by $\sim 25 \mathrm{~km} \mathrm{~s}^{-1}$ ) spread in velocity, consistent with the internal spread of MILES stars found in Prugniel et al. $\left(2011,12 \mathrm{~km} \mathrm{~s}^{-1}\right)$. The residual shifts and the Gaussian widths varies from star to star. At $5000 \AA$ the internal dispersion of the residual shift for the ELODIE comparison is $0.32 \AA$ (equivalent to an internal dispersion of $19 \mathrm{~km} \mathrm{~s}^{-1}$, or $\sim 0.1$ pixel), and the standard deviation of the FWHM broadening is $0.75 \AA$ (or $45 \mathrm{~km} \mathrm{~s}^{-1}$ ). The spread of the residual shift is similar to the the typical uncertainties from data reduction (usually 10 percent or lower). The variance of the FWHM width is partly physical (i.e. rotation) and partly instrumental (star-to-star difference of resolution).

\subsection{UVBlue theoretical grid}

The UVBLue library (Rodríguez-Merino et al. 2005) covers the wavelength range from $87 \mathrm{~nm}$ to $470 \mathrm{~nm}$ at $R=50000$. Its parameter coverage reaches from $T_{\text {eff }}=3000$ to $50000 \mathrm{~K}, \log g=$ 0.0 to 5.0 with steps of $0.5 \mathrm{dex}$, and $[\mathrm{Fe} / \mathrm{H}]=-2.0$ to $0.5 \mathrm{dex}$, computed at 7 nodes with a solar mix (Anders \& Grevesse 1989). The final grid consists of 1770 models with local thermodynamic equilibrium (LTE). These were computed using the updated list of atomic transition given by Kurucz (1992), adding all diatomic molecular lines except TiO. However, the latest molecule transitions are only prominent in late-type stars with

\footnotetext{
3 For the interpolator based on MILES, Prugniel et al. (2011) used the first official version of the library (v.9.0), while for the LSF comparison we used the new 9.1 version, where the wavelength calibration of some stars was re-done.

${ }^{4}$ Note that in some cases the distribution is very skewed, therefore even with clipping the mean may not coincide with the peak of the distribution.
}

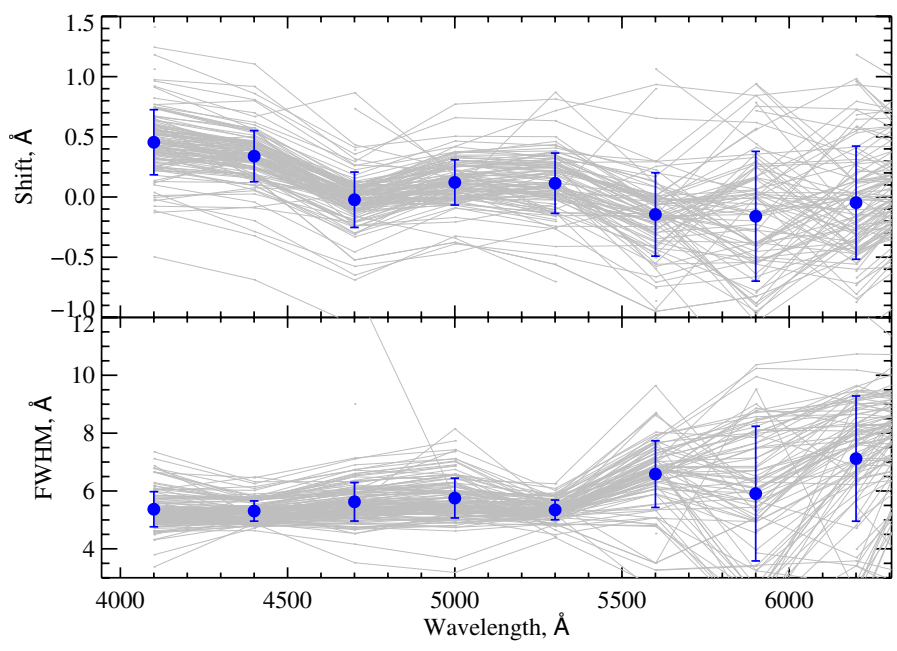

Fig. 1. Relative LSF for the 127 NGSL stars in common with the ELODIE library. The panels show the shift in wavelength and the resolution (FWHM) as a fucntion of wavelength. The obtained LSFs for the individual stars are plotted in thin grey lines. The mean values and their standard deviations are plotted in blue.

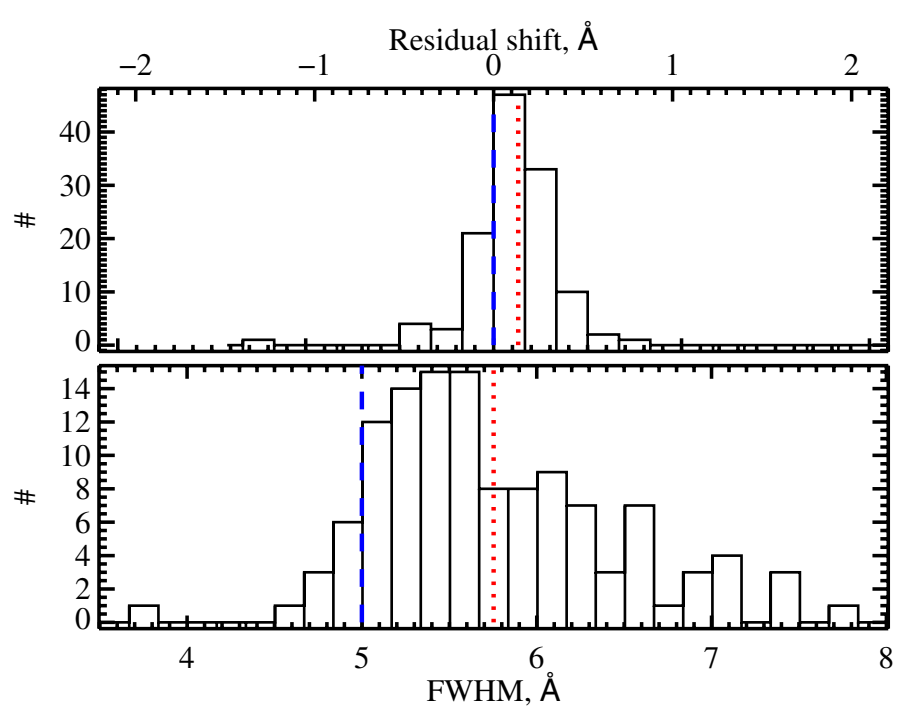

Fig. 2. Histograms corresponding to the distributions of the wavelength shift and the resolution at $\sim 5000 \AA$ for the 127 NGSL stars in common with ELODIE. The blue dashed vertical lines mark the zero velocity and the expected dispersion of $5 \AA$, while the red dotted lines are the mean residual shift and the mean FWHM (top and bottom respectively).

$T_{\text {eff }}$ bellow $4000 \mathrm{~K}$, which in turn do not have significant UV flux. The microturbulent velocity was fixed $\left(\xi=2 \mathrm{~km} \mathrm{~s}^{-1}\right.$, default for the Kurucz models). This value is expected to vary in the different spectroscopic classes from 1.5 to $10 \mathrm{~km} \mathrm{~s}^{-1}$, but the effects are expected to be negligible at low resolution (as in the present case). We downloaded the $R=10000$ version available online, calibrated in air wavelength. We measured its intrinsic resolution using the solar spectrum from the BASS2000 database ${ }^{6}$ and found it to be consistent with the value given by the authors.

Each of the NGSL stars was fitted against a positive linear combination of UVBlue spectra according to Eq. (3). For each

\footnotetext{
5 http://www.inaoep.mx/ modelos/uvblue/uvblue.html

${ }^{6}$ http://bass2000.obspm.fr/solar_spect.php
} 
NGSL stellar spectrum the comparison was made with the eight UVBlue spectra whose parameters surround the values from Table 1 (determined in Sect.5). We determined the weights of the different UVBlue spectra from a fit over the whole wavelength range, and we used this combination to analyse the individual segments. This first fit of the whole spectral range was also used to clip the spikes. We performed the LSF analysis again in the $400 \AA$ segments and we averaged the individual LSFs.

While fitting the LSF we noticed that the region between 2400 and $3000 \AA$ is poorly matched by the theoretical spectra. This discrepancy was already pointed by the authors of UVBlue (Rodríguez-Merino et al. 2005), stating that the simulated FGK stars fail to reproduce important prominent metallic features as the FeII blend at $2400 \AA$, FeI/Sir blend at about $2500 \AA$, MgII doublet at $2800 \AA \mathrm{Mg}$ line at $2852 \AA$ and the Mg break at $\sim 2600 \AA$. We find that the match is also poor for the OBA and M spectral types (although the low flux of the M stars in the blue reduces the significance of the comparisons because of the $\mathrm{S} / \mathrm{N}$ limitation). However, even though that many prominent features are misfitted, there is enough information in the other lines to constrain the LSF, but of course with a lower precision. Hence, the LSF determined in this region needs to be taken cum grano salis.

\subsection{Munari et al. theoretical grid}

To assess the LSF in the NIR part of the NGSL stars, we downloaded the $1 \AA / \mathrm{px}$, scaled solar version of the Munari library ${ }^{7}$ $(\lambda \lambda=2500-10500 \AA$, Munari et al. 2005). The library of 51288 spectra was produced using Kurucz models covering the space of atmospheric parameters as follows: $3500 \leq T_{\mathrm{eff}} \leq$ $47500 \mathrm{~K}, 0.0 \leq \log g \leq 5.0,-2.5 \leq[\mathrm{M} / \mathrm{H}] \leq 0.5,[\alpha / \mathrm{Fe}]=$ $0.0,+0.4, \xi=1,2,4 \mathrm{~km} \mathrm{~s}^{-1}, 0 \leq V_{\text {rot }} \leq 500 \mathrm{~km} \mathrm{~s}^{-1}$. They used the updated list of atomic transition by Kurucz (1992), adding some molecular lines, including TiO for stars with $T_{\text {eff }}<5000 \mathrm{~K}$. The authors compared the colours and temperatures of their predictions to other synthetic libraries and real stars, noticing that their models fail to reproduce the very red colour observed in low-temperature stars.

We measured its intrinsic resolution using the solar spectrum from the BASS2000 database and found it to be FWHM $=2.1 \AA$ throughout the full wavelength range. Again, the spectra are airwavelength-calibrated. The Munari grid was generated at high resolution, then convolved with a Gaussian to lower resolution and finally rebinned to pixels of half the FWHM of this Gaussian. Since the rebinning also implies a convolution by a top-hat function of 1 pixel, the final broadening is slightly larger than the convolving Gaussian. We performed the LSF analyses over the full wavelength range in the same way as for UVBlue, following Eq. (3).

\subsection{Corrected wavelength calibration and adopted LSF}

The LSFs obtained with the four reference libraries are represented in Fig. 3. The results from these comparisons are fully consistent.

The FWHM of the LSF is varying from $3 \AA$ at the UV end to $5 \AA$ at $5000 \AA$, and to $10 \AA$ at the NIR end. It is roughly constant over each segment corresponding to the different grat-

\footnotetext{
${ }^{7}$ http://archives.pd.astro.it/2500-10\protect $\backslash$ kern+. 1667 em $\backslash$ relax500/
}
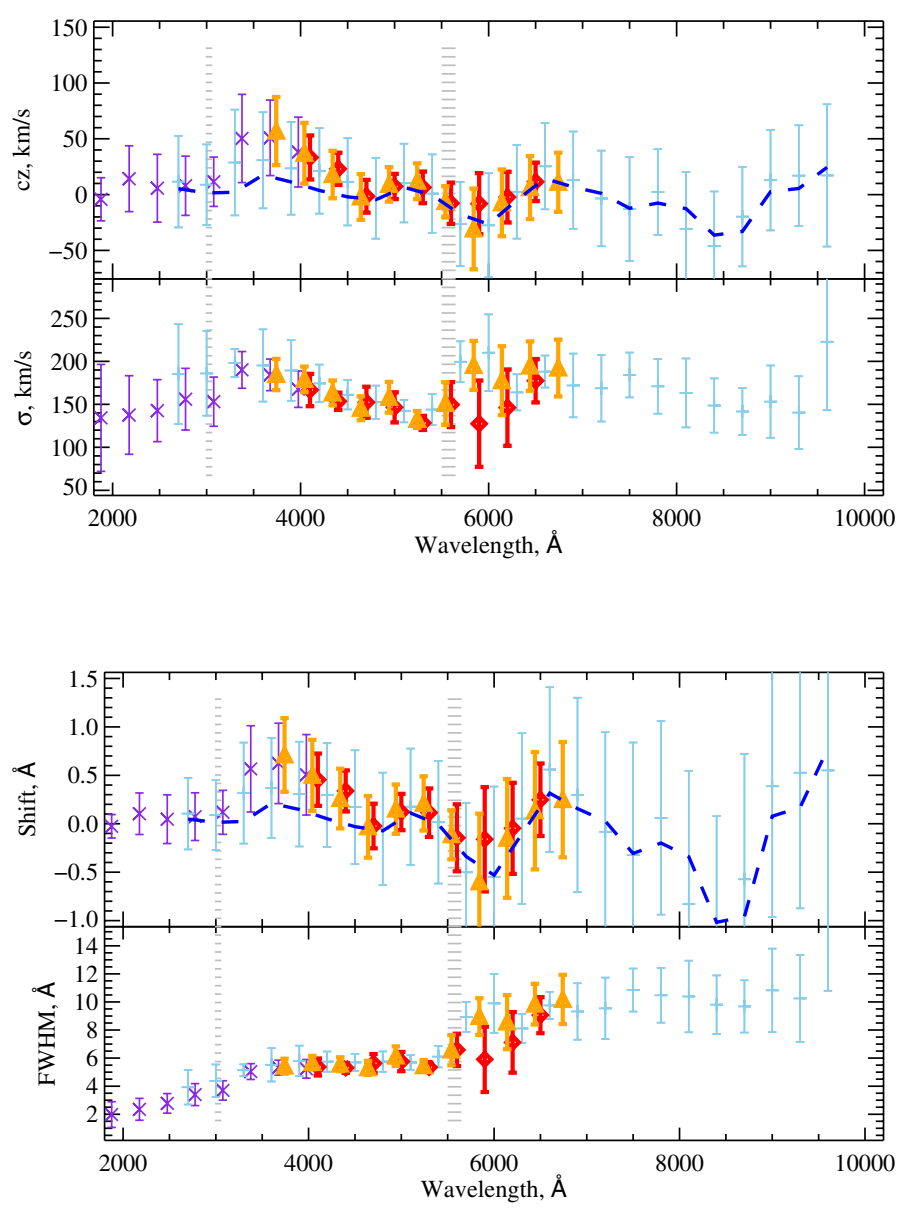

Fig. 3. Global line-spread function of NGSL. The upper panels show the residual velocity and instrumental velocity dispersion in $\mathrm{km} \mathrm{s}^{-1}$. The bottom panels show the corresponding wavelength shift and resolution in $\AA$. The LSFs obtained with the aid of the various reference libraries are overplotted with the symbols according to the legend in the first panel. The error bars represent the standard deviations from the distribution of the LSF obtained with the individual stars. The regions where the three segments of NGSL overlap are marked with grey horizontal lines. The blue dashed line shows the applied correction to the velocity shift. (MILES and ELODIE libraries have different starting points, therefore their results appear slightly shifted.)

ings, and the discontinuities between the three gratings, at 3060 and $5650 \AA$, are clear.

The residual shifts for the UV and red gratings do not significantly depend on the wavelength and are small: 10 and $0 \mathrm{~km} \mathrm{~s}^{-1}$. Our analysis reveals a defect of the wavelength calibration of the green segment (G430L grating). We used a simple linear relation to correct it: $\lambda_{\text {cor }}=\lambda-0.7(5650-\lambda) /(5650-3060)$, for $3060<\lambda<5650 \AA$, where $\lambda$ is the original wavelength in $\AA$ and $\lambda_{\text {cor }}$ the corrected wavelength. This first-order correction of the dispersion relation is derived from the drift of the residual wavelength shift seen on Fig. 3. This correction can easily be applied to the wavelength array when the NGSL FITS files are read.

We averaged the four LSFs and restored the discontinuities, which were smoothed by our analysis in $400 \AA$ segments by extrapolating the trend seen for each grating towards the overlap regions. 


\section{Atmospheric parameters}

We determined the atmospheric parameters of the NGSL stars by fitting the spectra against a reference spectrum of given $T_{\text {eff }}$, $\log g$ and $[\mathrm{Fe} / \mathrm{H}]$. We compared the parameters with various previous studies to assess their reliability and precision.

\subsection{Measurement of the parameters}

We determined the atmospheric parameters of NGSL with ULySS as in Wu et al. (2011b), Prugniel et al. (2011), and Wu et al. (2011a). The fit is performed according to Eq. (4) over the wavelength range 3500 to $7500 \AA$ (the wavelength range of the MILES stellar library). The spectra were logarithmically rebined to pixels corresponding to $100 \mathrm{~km} \mathrm{~s}^{-1}$. We used the LSF derived in the previous section as described in Koleva et al. (2009). Because of the variation of the LSF throughout the library, the convolution by $G$ in Eq. (4) was maintained. To prevent that the model adapted to the mean NGSL resolution becomes broader than a given spectrum, we biased the injected LSF by $100 \mathrm{~km} \mathrm{~s}^{-1}$ (i.e. we subtracted $100 \mathrm{~km} \mathrm{~s}^{-1}$ in quadrature from $\sigma_{\text {ins }}(\lambda)$ ). We used a 12 th degree multiplicative polynomial to absorb any continuum mismatches between the model and the observations (Sect. 3).

ULySS performs a local minimisation starting from a guess point in the parameter space. Thus, the solution may be trapped in a local minimum. To avoid this and reject local minima, we repeated the minimisation from multiple guesses, sampling the parameters spaces at the following nodes: $T_{\text {eff }}$ in $[3500,4000,5600,7000,10000,18000,30000],[\mathrm{Fe} / \mathrm{H}]$ in $[-1.7,-0.3,0.5]$ and $\log g$ in $[1.8,3.8]$.

Previous studies, in particular Wu et al. (2011b), have shown that while this method is highly reliable for FGK stars, special care has to be taken for OBA and M stars. There are various reasons for this lower reliability: First, the reference library is more scarcely populated in these regions of the parameters space; second, high-precisions measurements of the atmospheric parameters of these stars are not as abundant as for FGK stars; finally, the determination of the parameters of those stars is more complex for physical reasons. Therefore, we systematically checked our determinations against recent literature for OBA and $\mathrm{M}$ stars as well as for cases that we found in disagreement with other studies. For 30 stars (8 percent of the library) we adopted measurements from the literature. Our final list of parameters are given in Table 2. The distribution of the stars in $T_{\text {eff }}-\log g$ and $T_{\text {eff }}-[\mathrm{Fe} / \mathrm{H}]$ planes is presented in Fig. 4.

\subsection{Comparison with the literature}

We compared our measured parameters with those from four previous studies by Heap \& Lindler (2009), Wu et al. (2011b), Prugniel et al. (2011), and Soubiran et al. (2010).

Heap \& Lindler (2009) derived preliminary, main atmospheric parameters of the NGSL (version 1). They derived $T_{\text {eff }}$ and the metallicity by fitting the stellar spectra to models of Munari (Munari et al. 2005). The gravity was obtained from the star position on the HR diagram using the Hipparcos parallax for the distances. The comparison of their results with the Valenti \& Fischer (2005) catalogue of cool stars shows some deviations for the $\log g$, which are not unexpected because the atmospheric parameters are coupled, and deriving them separately may lead to biases.
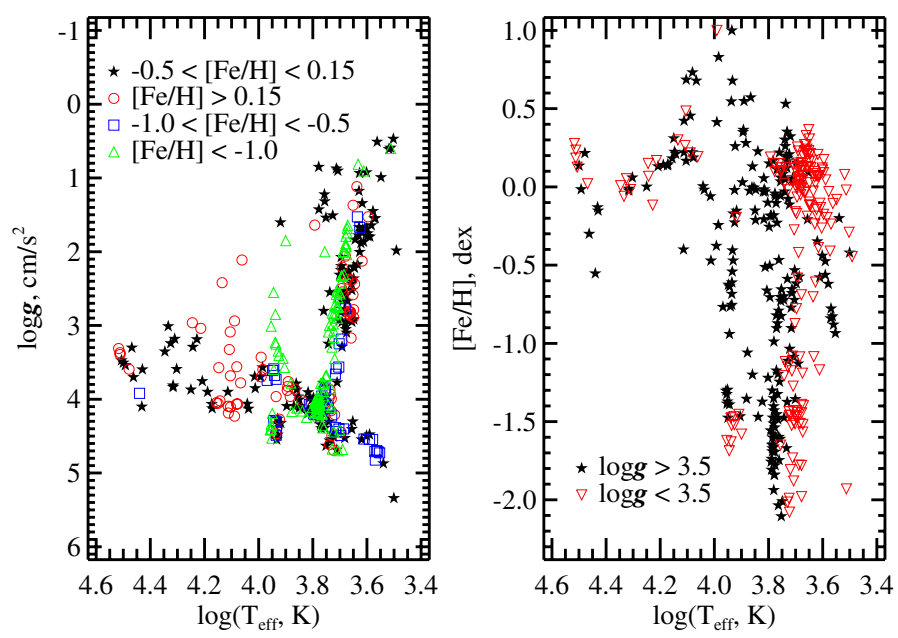

Fig. 4. Resulting stellar parameter coverage for the 374 stars of the NGSL. The left panel shows their distribution in the $T_{\text {eff }}-\log g$ plane. These stars were separated into four different metallicity bins according to the legend. In the right panel we plot the dwarf and giant distribution in the $T_{\text {eff }}-[\mathrm{Fe} / \mathrm{H}]$ plane.

The determination of the CFLIB and MILES parameters (Wu et al. 2011b; Prugniel et al. 2011) was performed as in the present paper. This method is highly reliable for intermediate spectral types, but it is not as accurate for the extremes of the HR diagram, as discussed in Sect. 5.1. There are, however, two main differences that may affect the comparison. On one hand, the presently analysed library has a much lower resolution than that of CFLIB $(R \sim 5000)$ and MILES $(R \sim 2000)$. The information from the weak lines accordingly is blended, which is particularly important when analysing earlier stellar types. On the other hand, the MILES interpolator, performs better than ELODIE (3.1 or 3.2) in some regions of the HR diagram (in particular for blue-horizontal branch stars, Prugniel et al. 2011). Hence, stars in this regions will possibly have a more precise determination of their atmospheric parameters.

Finally, the latest version of the PASTEL database (Soubiran et al. 2010) is a compilation of previously published stellar atmospheric parameters. It continues series of publications by Cayrel and collaborators (Cayrel de Strobel et al. 2001). Most of the measurements were made using high spectral resolution and high signal-to-noise data, though inhomogeneous. When there were multiple measurements of the same parameter of a given star available, we took the mean value.

The statistics of the comparisons was made without the outliers and it is presented in Table 1. The corresponding figures with the four comparison are given in Appendix B.

The FGK stars agree well with the results of the other authors. The biases are within the errors of the individual parameters and are not significant.

The linear fit of the comparison has slopes $(\nabla)$ close to unity in almost all cases. The dispersions from the comparisons are higher than in Prugniel et al. (2011), revealing a lower precision, which is probably caused by the lower spectral resolution.

The strongest discrepancy for the FGK spectral classes appears when comparing our $\log g$ values with that derived by Heap \& Lindler (2009) from the same data (see Fig. B.1). Our gravities are up to one dex higher for the dwarfs. This is consistent with the bias found in Heap \& Lindler (2009) by comparing to Valenti \& Fischer (2005). The discretisation of the measurements of Heap \& Lindler (2009) on the nodes of the model's grid 
Table 1. Comparison of the atmospheric parameters with other studies.

\begin{tabular}{l|cc|ccc|ccc|ccc}
\hline \hline \multirow{2}{*}{ Comparison } & \multicolumn{2}{|c|}{$N^{a}$} & \multicolumn{3}{c|}{$T_{\text {eff }}^{b}$} & \multicolumn{3}{c|}{$\log g\left(\mathrm{~cm} \mathrm{~s}^{-2}\right)$} & \multicolumn{3}{c}{ [Fe/H] (dex) } \\
& & & $\Delta$ & $\sigma$ & $\nabla$ & $\Delta$ & $\sigma$ & $\nabla$ & $\Delta$ & $\sigma$ & $\nabla$ \\
\hline \multirow{3}{*}{ NGSL } & OBA & 59 & 4 & 3 & 0.87 & 0.12 & 0.47 & 0.45 & -0.10 & 0.60 & 0.56 \\
& FGK & 236 & 4 & 171 & 1.08 & 0.22 & 0.42 & 1.18 & 0.09 & 0.34 & 0.94 \\
& M & 6 & -42 & 120 & 1.14 & 0.32 & 0.55 & 1.32 & -0.35 & 0.40 & -0.05 \\
\hline \multirow{3}{*}{ MILES } & OBA & 20 & 6 & 6 & 0.96 & 0.11 & 0.45 & 0.87 & -0.02 & 0.19 & 0.99 \\
& FGK & 55 & 26 & 115 & 1.01 & 0.03 & 0.28 & 1.10 & -0.00 & 0.11 & 1.01 \\
& M & 4 & 41 & 37 & 1.02 & 0.13 & 0.14 & 0.83 & 0.08 & 0.19 & 0.06 \\
\hline \multirow{3}{*}{ CFLIB } & OBA & 32 & 5 & 3 & 1.04 & 0.05 & 0.30 & 0.93 & 0.03 & 0.22 & 1.03 \\
& FGK & 80 & 43 & 111 & 1.00 & -0.00 & 0.20 & 1.04 & 0.04 & 0.11 & 1.01 \\
& M & 5 & 37 & 23 & 0.98 & 0.46 & 0.69 & 0.24 & 0.17 & 0.29 & 1.00 \\
\hline \multirow{3}{*}{ PASTEL } & OBA & 57 & 7 & 6 & 1.06 & 0.06 & 0.43 & 0.65 & -0.00 & 0.63 & 0.90 \\
& FGK & 176 & 69 & 196 & 1.00 & 0.04 & 0.42 & 1.09 & 0.05 & 0.19 & 1.03 \\
& $\mathrm{M}$ & 5 & -73 & 246 & 0.51 & -0.13 & 0.19 & 1.02 & -0.26 & 0.33 & 0.60 \\
\hline
\end{tabular}

Notes. For each parameter the $\Delta$ column gives the mean difference "this work" - "reference", $\sigma$ the dispersion between the two series and $\nabla$ the slope from the linear fit. The three lines are for the OBA $\left(T_{\text {eff }}>8000 \mathrm{~K}\right)$, FGK $\left(4000<T_{\text {eff }} \leq 8000 \mathrm{~K}\right)$ and M $\left(T_{\text {eff }} \leq 4000 \mathrm{~K}\right)$ spectroscopic types, respectively. The statistics were computed discarding the outliers. ${ }^{(a)}$ Number of compared spectra ${ }^{(b)}$ The $\Delta$ and $\sigma$ of $T_{\text {eff }}$ are in K, except for the OBA stars, where these statistics are given in percent.

is also apparent in the figure. This is because the authors did not interpolate between the templates.

The automatic determination of the parameters is more difficult for OBA and M stars. At this low resolution only few lines are present for early-type stars, and the parameters are less constrained. In addition, the profiles of the lines are also affected by rotation (and inclination of the rotation axis on the line-of-sight) and some stars display emission lines. For the late types, on the other hand, the spectra are dominated by broad molecular features and the individual narrow band is lost because of the low resolution. Despite these difficulties, the comparison with previous studies revealed no trend or bias.

To summarise, our results are consistent with those of authors for all spectral types. The offsets in $T_{\text {eff }}$ vary from 4 to $69 \mathrm{~K}$ in the FGK stars, depending on the comparison study with a dispersion of $\sim 150 \mathrm{~K}$; in $\log g$ the offset found in comparison with Heap \& Lindler (2009) is 0.22 , while there is no offset with the other references, the typical dispersion is about 0.35 dex; the shift in metallicity is negligible and the dispersions are between 0.11 to 0.34 dex. For the OBA spectral types we find $\Delta\left(T_{\text {eff }}, K\right) \sim 5 \%, 0.05<\Delta\left(\log g, \mathrm{~cm} / \mathrm{s}^{2}\right)<0.12$, with a dispersion $\sim 0.47$ and $-0.02<\Delta[\mathrm{Fe} / \mathrm{H}]$, dex $<0.10$, varying between 0.2 and 0.6 dex. There are too few $\mathrm{M}$ stars in common between the different data sets to make any statistical analyses.

\subsection{Error estimation}

The errors returned by ULySS are computed from the covariance matrix. They underestimate the real precision because (i) the parameters are not fully independent (for example there is the well-known degeneracy between $T_{\text {eff }}$ and $\log g$ ); (ii) the fits are not perfect (there are some mismatches caused by non-solar abundances or particularities); and (iii) the errors in the data are not accurately known. Therefore, we first determined an upper limit to the internal error by forcing $\chi^{2}=1$, and we estimated the external error by rescaling the internal error as in $\mathrm{Wu}$ et al. (2011b).

To estimate the external errors we used the statistics of the comparison between our determination and Prugniel et al. (2011, see Sect. 5.2). We chose this reference for the comparison because it is homogeneous and reasonably accurate. The external errors were determined in Prugniel et al., therefore we subtracted them quadratically from the dispersion of the comparison and obtained an estimate of the mean external error. From this mean external error we derived the rescaling factor. For a given spectral type and stellar parameter, the rescaling factor is computed as

$\xi=\frac{\sqrt{\sigma_{\text {tot }}^{2}-\bar{\sigma}_{\mathrm{mil}}^{2}}}{\bar{\sigma}_{\mathrm{int}}}$

where $\sigma_{\text {tot }}$ is the residual dispersion from corresponding comparison in Table $1, \bar{\sigma}_{\text {mil }}$ the external errors reported in Prugniel et al. (2011), and $\overline{\sigma_{\text {int }}^{-}}$the mean internal error from the present fits. The final corrected error for each of the stars is $\sigma_{i}^{\prime}=\xi \times \sigma_{i}$.

The correction coefficients are about 2.5 for the FGK stars. For the early spectroscopic classes they vary from 2.5 (for metallicity) to 6.0 (for gravity). We do not have sufficiently high statistics to compute these coefficients for the M class. However, we consider that the external errors are roughly the same as for the OBA stars and as in Prugniel et al. (2011). Therefore, we used their coefficients to correct the errors of $\mathrm{M}$ stars. The median precision of the derived parameters is $42 \mathrm{~K}$ for $T_{\text {eff }}, 0.24$ dex in $\log g$ and 0.09 dex in $[\mathrm{Fe} / \mathrm{H}]$ for the FGK class. For the OBA stars they are 4.5 percent, 0.44 dex and $0.18 \mathrm{dex}$, and for the $\mathrm{M}$ stars $29 \mathrm{~K} 0.50 \mathrm{dex}$ and $0.48 \mathrm{dex}$ for temperature, gravity, and metallicity, respectively. The precisions are lower than those obtained by Prugniel et al. (2011), probably because of the lower spectral resolution. 


\section{Galactic extinction}

The Galactic extinction may be determined from photometry (e.g. Neckel \& Klare 1980) or using a Galactic model (Chen et al. 1998; Hakkila et al. 1997). The first method requires (i) accurate photometry and (ii) a good knowledge of the intrinsic SED of the stars (i.e. a precise spectral classification). A wrong estimate of the metallicity will immediately translate into an error on the Galactic extinction. The second method requires knowledge of the direction and distance to the star and an adequate model of the Galaxy. For example the Chen et al. (1998) model is a simple geometric representation of the galaxy scaled to the "total" extinction provided by the Schlegel maps. Alternatively the Hakkila et al. (1997) model is calibrated on empirically measured extinctions in several directions in the galaxies. These models are generally acceptable in low-extinction regions (i.e. Galactic latitude $|b|>10^{\circ}$ ), but they are less reliable in high-extinction regions.

An alternative to these two methods is to directly measure the extinction on the NGSL spectra. The NGSL spectra were flux-calibrated with a precision of 2-3 percent (Heap \& Lindler 2009). Therefore, $P_{n}$ mixes information about the uncertainty of the flux calibration and the Galactic extinction. Hence, we can assume to first approximation that $P_{n}$ (Eq. (4)) derived in Sect. 5 corresponds to the extinction curve. We therefore fitted $P_{n}$ against the Galactic extinction law from Fitzpatrick (1999). The precision on the derived $E(B-V)$ colour excess depends on the precision on (i) the flux calibration, (ii) the atmospheric parameters, and (iii) the best-fitted template. The precision of the ELODIE and MILES interpolators were discussed in Prugniel et al. (2011) and were found to be accurate to 1-2 percent.

The extinction law, $A(\lambda) / A_{V}$ (normalised to the $V$ band) depends on the line-of-sight. It can be parameterised with $R_{V}=$ $A_{V} / E(B-V)$ (the ratio between the extinction in the $V$ and the $B$ $V$ colour excess), which have a "mean" value of 3.1, but it varies between 2.3 and 5.3 (Cardelli et al. 1989; Fitzpatrick 1999). The extinction is almost independent of $R_{V}$ in the red, but is strongly dependent on the wavelength in the blue and UV. Adopting the Fitzpatrick (1999) extinction law, we were able to fit simultaneously $A_{V}$ and $R_{V}$ over almost the full wavelength range by comparing the observed spectra to the Munari best fit. However, it is known that the SED of the theoretical spectra might not provide a good match to the empirical counterpart, particularly in the shortest wavelengths. For this reason, we preferred to compare the observations to the best-fitted interpolated MILES spectrum obtained in Sect. 5.

Fitting the extinction using MILES restricts the wavelength range to the optical domain, and therefore the correction of the whole spectrum requires extrapolations. The extrapolation towards the infrared should be safe because the extinction law is uniform in any line-of-sight (and the extinction is low), and the quality of the corrected spectrum will be essentially limited by the precision of the original flux calibration. However, the extrapolation towards the UV can be more hazardous because the determination of $R_{\mathrm{V}}$ will only rely on the blue end of the MILES spectra. Any error on the extinction law would be amplified in the UV. For this reason, we preferred here to adopt $R_{V}=3.1$ and we fitted only $A_{V}$ over the wavelength range 3500-7500.

To test the reliability of these determinations of the extinction, we compared them with the predictions of the Chen et al. (1998) Galactic extinction model for the stars with parallaxes known from Hipparcos ${ }^{8}$. This comparison is acceptable with a slope of 0.85 . Our values of $A_{V}$ are listed in Table 1.

\section{Conclusions}

We have fully characterised the NGSL for its implementation in stellar population synthesis modelling. We used ULySS, a full spectrum fitting package. We found that the line-spread function of the stellar spectra of this library vary from $3 \AA$ in the UV to $10 \AA$ (FWHM) in the near IR. The instrumental velocity dispersion is virtually constant within the whole spectral range covered by the library, at $\sigma_{\text {ins }} \approx 130 \mathrm{~km} \mathrm{~s}^{-1}$. The wavelength calibration is accurate to $0.1 \mathrm{px}(0.32 \AA$ at $5000 \AA)$. We measured the atmospheric parameters of the stars using the ULySS package and the MILES interpolator Prugniel et al. (2011). By comparing the results to previous studies we found that the precisions for the FGK stars are $42 \mathrm{~K}, 0.24$ and 0.09 dex for $T_{\text {eff }}, \log g$ and $[\mathrm{Fe} / \mathrm{H}]$, respectively. For the $\mathrm{M}$ stars, the corresponding mean errors are $29 \mathrm{~K}, 0.50$ and $0.48 \mathrm{dex}$, and for the OBA 4.5 percent, 0.44 and 0.18 dex. Finally, we measured the Galactic extinction for each star by directly comparing the spectra to the interpolated MILES spectra.

The NGSL library is a major step towards the accurate modelling of stellar populations over a wide wavelength range. In the second paper of this series we make use of the NGSL and the results of this work to expand the spectral coverage of our stellar population models (Vazdekis et al. 2010).

Acknowledgements. This work has been supported by the Programa Nacional de Astronomía y Astrofísica of the Spanish Ministry of Science and Innovation under grant AYA2010-21322-C03-02. M.K. thanks CRAL, Observatoire de Lyon, Université Claude Bernard for an Invited Professorship and Ph. Prugniel for the useful comments. She is postdoctoral fellow of the Fund for Scientific ResearchFlanders, Belgium (FWO11/PDO/147) and Marie Curie (Grant PIEF- GA-2010271780) fellow We would like to thank S. Heap and the referee for their helpful comments.

\section{Appendix A: Atmospheric parameters}

Here we list the adopted parameters of the 367 NGSL stars (Table A1), together with the extinction in $V$, the values of the $\mathrm{S} / \mathrm{N}$ at 3 different wavelengths, roughly corresponding to the middle of the range from the blue, green, and red arm of the STIS spectrograph. There are 35 stars that SIMBAD recognises as spectroscopic binaries, they are marked with a star (*). Finally, in this table we give the references for the stars with parameters adopted from the literature.

\section{Appendix B: Comparison with other libraries}

Here we plot the comparisons with the literature discussed in Sect. 5.2. For each of the reference libraries we plot the different spectroscopic classes in different panels. We omit the M class because there are too few (14) cold stars in NGSL. We provide the usual "our" vs. "literature" value plots, but we also investigate how the residuals of this comparison depend on the different parameters.

\footnotetext{
8 http://www.rssd.esa. int/index.php? project $=$ HIPPARCOS\&page $=$ index
} 
M. Koleva and A. Vazdekis: Stellar atmospheric parameters for NGSL. I.

95 OBA stars
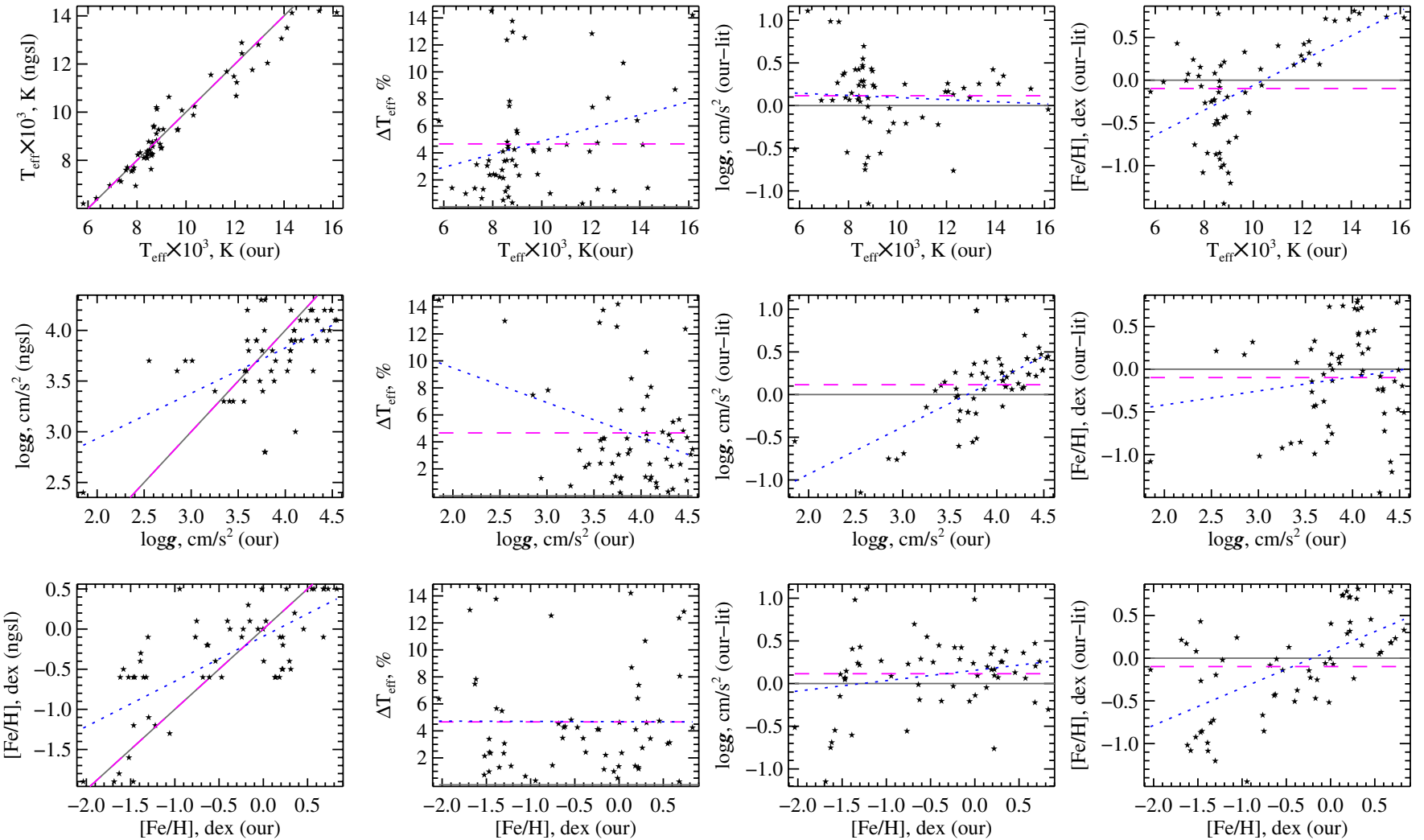

253 FGK stars
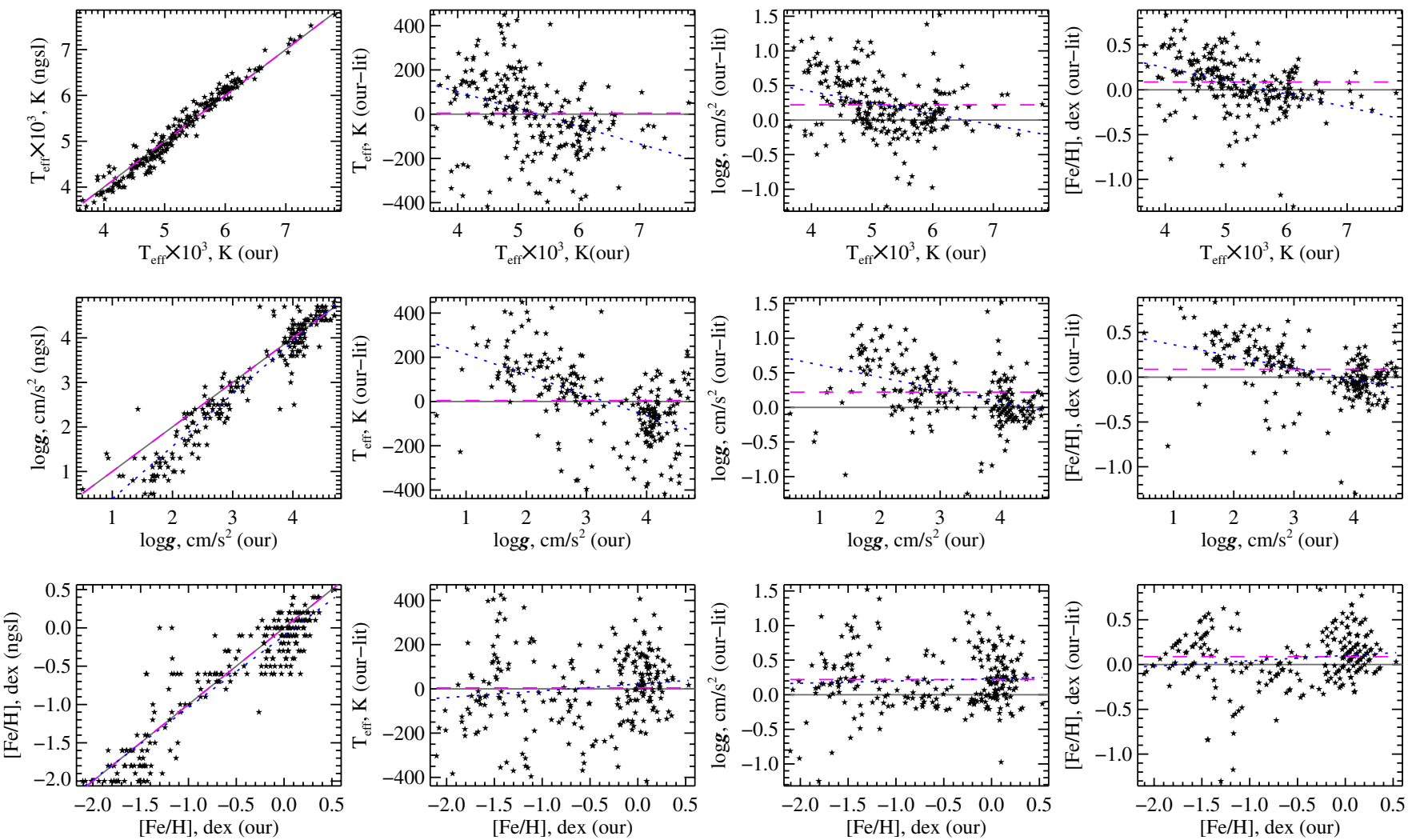

Fig. B.1. Comparison of the original stellar atmospheric parameters published for the NGSL with those derived here using ULySS and the MILES interpolator. The median value of the residuals are plotted in magenta-dashed lines, while the fit to the residuals are plotted as blue-dotted lines. The one-to-one relation is shown in black. The upper three rows of panels show the results for the OBA stars. The remaining sets of panels show the results for the FGK stars. For each set of stellar spectral types we show in the first column of panels the comparison of the temperature, gravity, and metallicity values from top to bottom. In the last three columns of panels we show the residuals in temperature, gravity, and metallicty (from left to right) as a function of temperature, gravity, and metallicity (from top to bottom). 


\section{OBA stars}
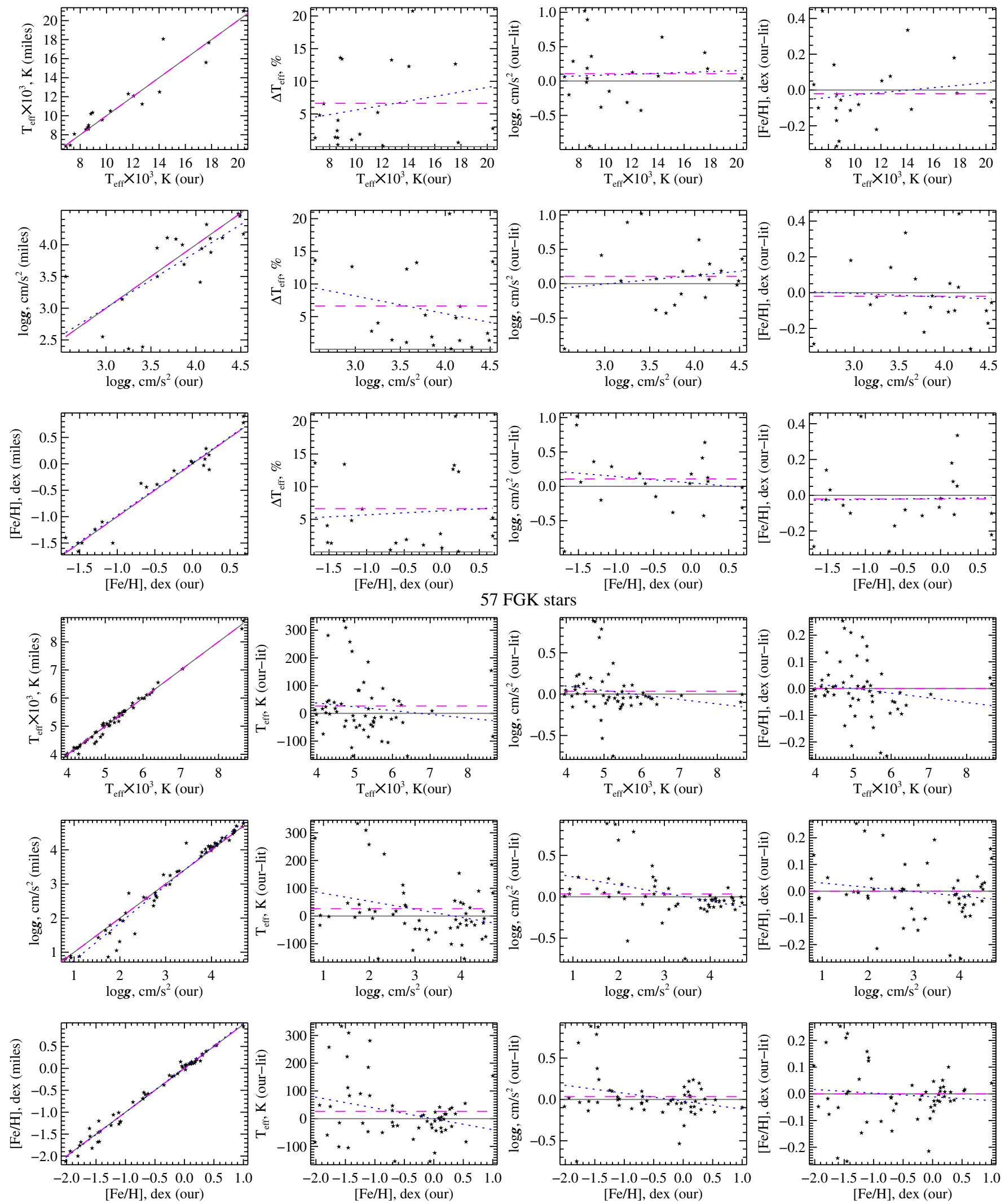

Fig. B.2. Comparison of the stellar parameters derived in this work with those from Prugniel et al. (2011) on the basis of the stars in common between these two libraries. Points and line types as in Fig. B.1. 
M. Koleva and A. Vazdekis: Stellar atmospheric parameters for NGSL. I.

38 OBA stars
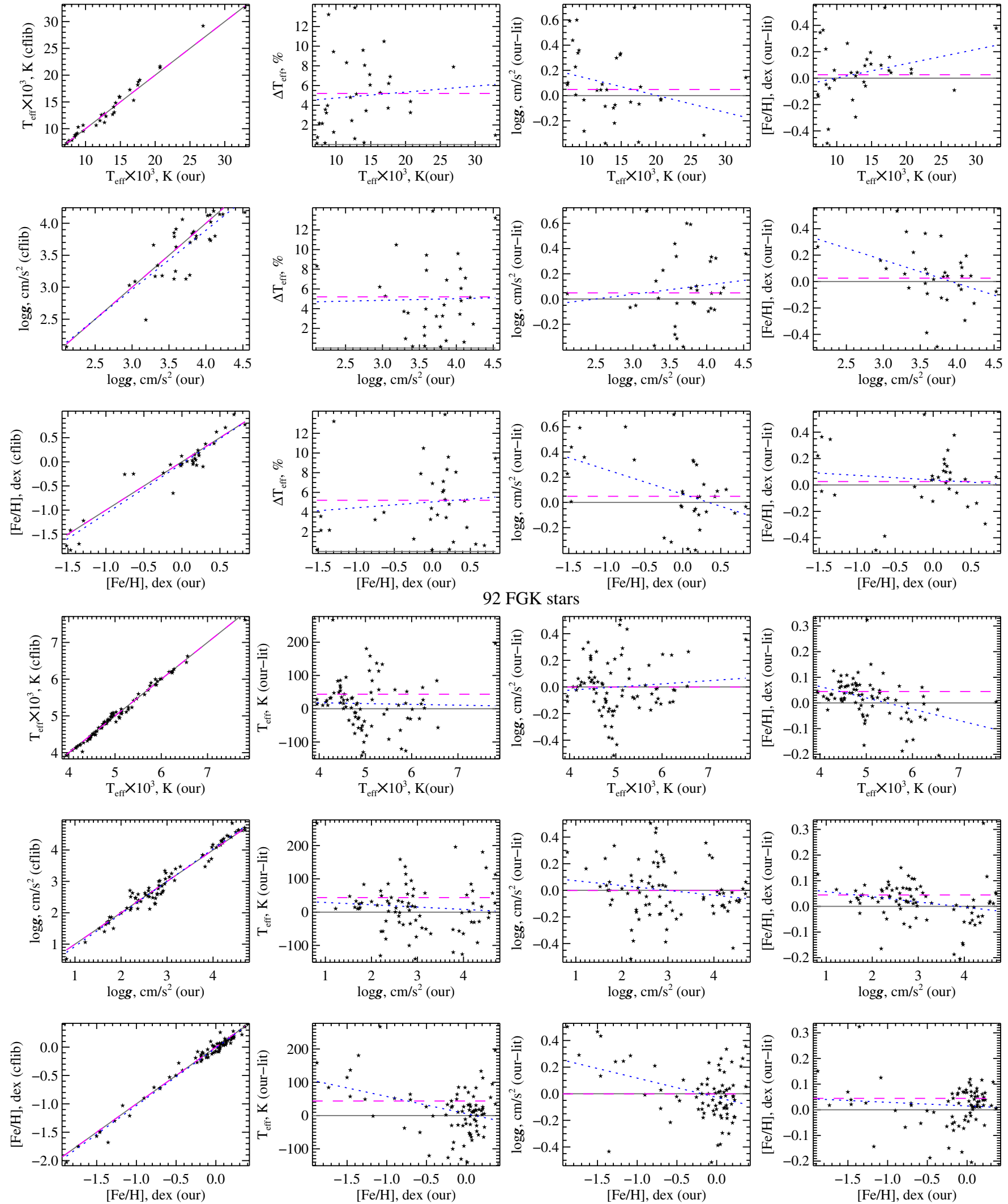

Fig. B.3. Comparison of the stellar parameters derived in this work with those from Wu et al. (2011b) on the basis of the stars in common between these two libraries. Points and line types as in Fig. B.1. 
69 OBA stars
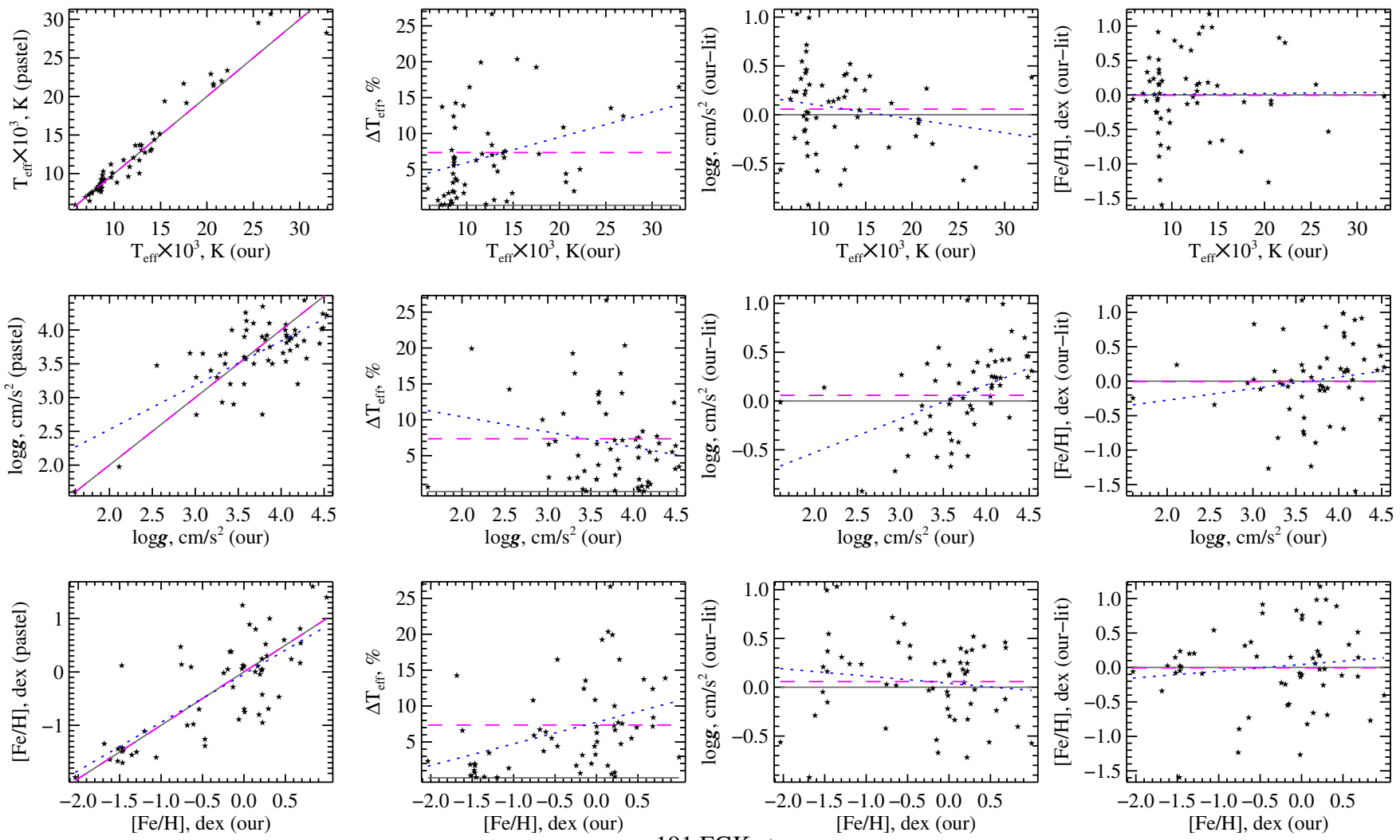

191 FGK stars
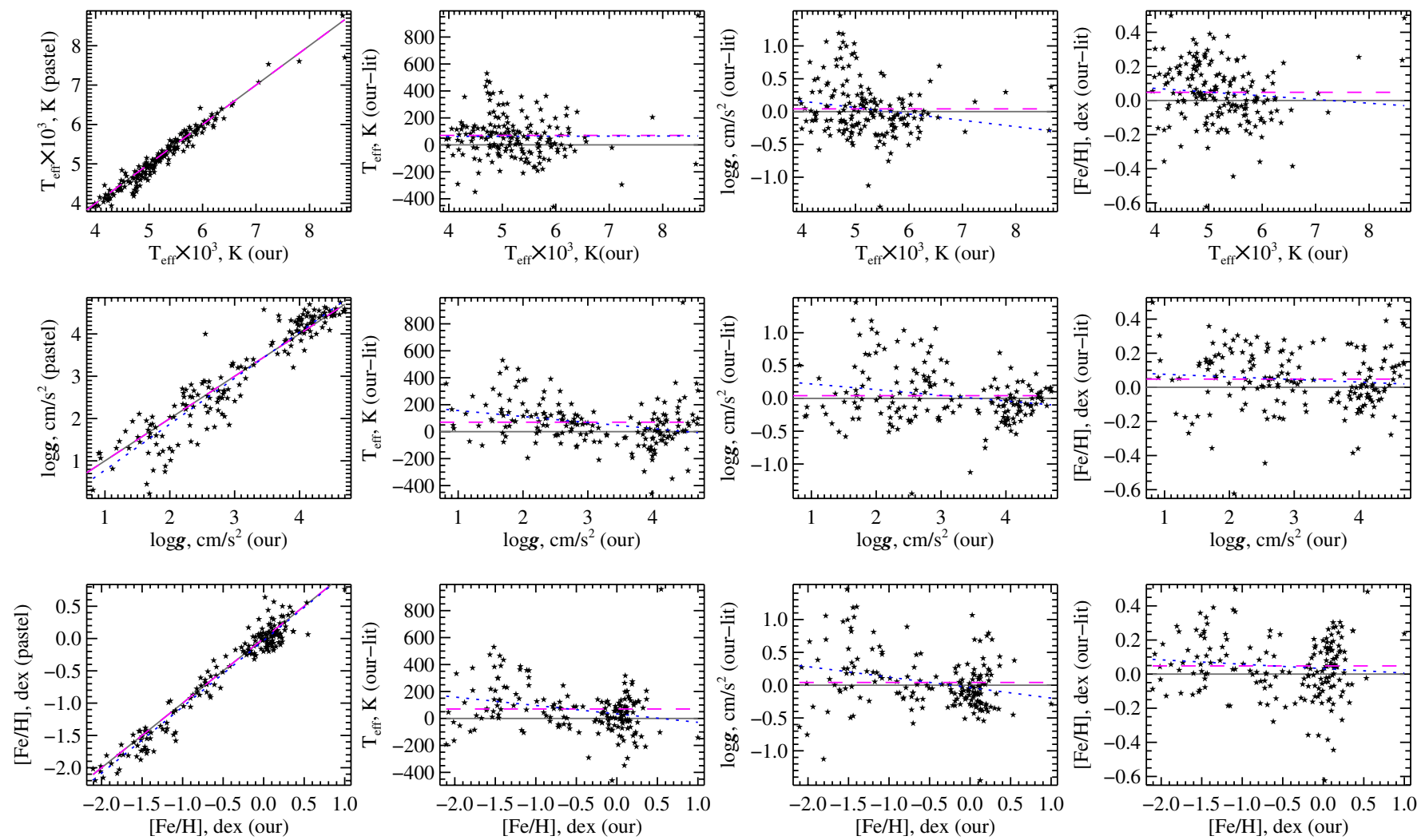

Fig. B.4. Comparison of the stellar parameters derived in this work with those from Pastel database on the basis of the stars in common between these two libraries. Points and line types as in Fig. B.1. 
M. Koleva and A. Vazdekis: Stellar atmospheric parameters for NGSL. I.

\section{References}

Adelman, S. J., \& Pintado, O. I. 2000, A\&A, 354, 899

Anders, E., \& Grevesse, N. 1989, Geochim. Cosmochim. Acta, 53, 197

Behr, B. B. 2003, ApJS, 149, 101

Beifiori, A., Maraston, C., Thomas, D., \& Johansson, J. 2011, A\&A, 531, A109

Bessell, M. S. 2005, ARA\&A, 43, 293

Bonfils, X., Delfosse, X., Udry, S., et al. 2005, A\&A, 442, 635

Bruzual A., G. 2007, A\&A, 419, 725

Cappellari, M., \& Emsellem, E. 2004, PASP, 116, 138

Cardelli, J. A., Clayton, G. C., \& Mathis, J. S. 1989, ApJ, 345, 245

Cassisi, S., Salaris, M., Castelli, F., \& Pietrinferni, A. 2004, ApJ, 616, 498

Castelli, F., \& Kurucz, R. L. 2004, [arXiv:astro-ph/0405087]

Cayrel de Strobel, G., Soubiran, C., \& Ralite, N. 2001, A\&A, 373, 159

Cenarro, A. J., Cervantes, J. L., Beasley, M. A., Marín-Franch, A., \& Vazdekis, A. 2008, ApJ, 689, L29

Cervantes, J. L., Coelho, P., Barbuy, B., \& Vazdekis, A. 2007, in IAU Symp. 241, ed. A. Vazdekis, \& R. F. Peletier, 167

Chen, B., Vergely, J. L., Valette, B., \& Carraro, G. 1998, A\&A, 336, 137

Coelho, P., Barbuy, B., Meléndez, J., Schiavon, R. P., \& Castilho, B. V. 2005, A\&A, 443, 735

Faber, S. M. 1983, Highlights Astron., 6, 165

Falcón-Barroso, J., Sánchez-Blázquez, P., Vazdekis, A., et al. 2011, A\&A, 532, A95

Fanelli, M. N., O’Connell, R. W., Burstein, D., \& Wu, C.-C. 1992, ApJS, 82, 197

Fitzpatrick, E. L. 1999, PASP, 111, 63

For, B.-Q., \& Sneden, C. 2010, AJ, 140, 1694

Gray, R. O., Corbally, C. J., \& Philip, A. G. D. 1996, AJ, 112, 2291

Gregg, M. D., Silva, D., Rayner, J., et al. 2006, in The 2005 HST Calibration Workshop: Hubble After the Transition to Two-Gyro Mode, ed A. M. Koekemoer, P. Goudfrooij, \& L. L. Dressel, 209

Hakkila, J., Myers, J. M., Stidham, B. J., \& Hartmann, D. H. 1997, AJ, 114, 2043

Hauschildt, P. H., Allard, F., Baron, E., Aufdenberg, J., \& Schweitzer, A. 2003 in GAIA Spectroscopy: Science and Technology, ed. U. Munari, ASP Conf. Ser., 298, 179

Heap, S., \& Lindler, D. J. 2009, in New Quests in Stellar Astrophysics II. Ultraviolet Properties of Evolved Stellar Populations, ed. M. Chávez Dagostino, E. Bertone, D. Rosa Gonzalez, \& L. H. Rodriguez-Merino, 273

Heap, S. R., \& Lindler, D. 2010, in BAAS, Am. Astron. Soc. Meet. Abstracts \#215, 42, 463

Kinman, T., Castelli, F., Cacciari, C., et al. 2000, A\&A, 364, 102

Koleva, M., Prugniel, P., Ocvirk, P., Le Borgne, D., \& Soubiran, C. 2008, MNRAS, 385, 1998

Koleva, M., Prugniel, P., Bouchard, A., \& Wu, Y. 2009, A\&A, 501, 1269

Kovtyukh, V. V. 2007, MNRAS, 378, 617

Kurucz, R. L. 1979, ApJS, 40, 1

Kurucz, R. L. 1992, Mex. Astron. Astrofis., 23, 45
Lee, H.-c., Lee, Y.-W., \& Gibson, B. K. 2002, AJ, 124, 2664

Lyubimkov, L. S., Rachkovskaya, T. M., Rostopchin, S. I., \& Lambert, D. L. 2002, MNRAS, 333, 9

Lyubimkov, L. S., Lambert, D. L., Rostopchin, S. I., Rachkovskaya, T. M., \& Poklad, D. B. 2010, MNRAS, 402, 1369

Mapelli, M., Ripamonti, E., Tolstoy, E., et al. 2007, MNRAS, 380, 1127

Maraston, C., \& Thomas, D. 2000, ApJ, 541, 126

Martins, L. P., \& Coelho, P. 2007, MNRAS, 381, 1329

Morales, J. C., Ribas, I., \& Jordi, C. 2008, A\&A, 478, 507

Munari, U., Sordo, R., Castelli, F., \& Zwitter, T. 2005, A\&A, 442, 1127

Neckel, T., \& Klare, G. 1980, A\&AS, 42, 251

Ocvirk, P. 2010, ApJ, 709, 88

Palacios, A., Gebran, M., Josselin, E., et al. 2010, A\&A, 516, A13

Percival, S. M., \& Salaris, M. 2011, MNRAS, 412, 2445

Prugniel, P., \& Soubiran, C. 2001, A\&A, 369, 1048

Prugniel, P., Soubiran, C., Koleva, M., \& Le Borgne, D. 2007b, unpublished [arXiv:astro-ph/0703658]

Prugniel, P., Koleva, M., Ocvirk, P., Le Borgne, D., \& Soubiran, C. 2007a, in IAU Symp. 241, ed. A. Vazdekis, \& R. F. Peletier, 68

Prugniel, P., Vauglin, I., \& Koleva, M. 2011, A\&A, 531, A165

Rodríguez-Merino, L. H., Chavez, M., Bertone, E., \& Buzzoni, A. 2005, ApJ, 626,411

Rose, J. A. 1984, AJ, 89, 1238

Sánchez-Blázquez, P., Peletier, R. F., Jiménez-Vicente, J., et al. 2006, MNRAS, 371, 703

Schiavon, R. P., Rose, J. A., Courteau, S., \& MacArthur, L. A. 2004, ApJ, 608, L33

Serven, J., Worthey, G., Toloba, E., \& Sánchez-Blázquez, P. 2011, AJ, 141, 184 Soubiran, C., Le Campion, J.-F., Cayrel de Strobel, G., \& Caillo, A. 2010, A\&A, 515, A111

Takada-Hidai, M., Takeda, Y., Sato, S., et al. 2002, ApJ, 573, 614

Tonry, J., \& Davis, M. 1979, AJ, 84, 1511

Valdes, F., Gupta, R., Rose, J. A., Singh, H. P., \& Bell, D. J. 2004, ApJS, 152, 251

Valenti, J. A., \& Fischer, D. A. 2005, VizieR Online Data Catalog, 215, 90141

van Belle, G. T., Creech-Eakman, M. J., \& Hart, A. 2009, MNRAS, 394, 1925

Vazdekis, A., Sánchez-Blázquez, P., Falcón-Barroso, J., et al. 2010, MNRAS, 404,1639

Walcher, C. J., Coelho, P., Gallazzi, A., \& Charlot, S. 2009, MNRAS, 398, L44

Wheeler, J. C., Sneden, C., \& Truran, Jr., J. W. 1989, ARA\&A, 27, 279

Wu, C.-C., Ake, T. B., Boggess, A., et al. 1983, NASA IUE Newsl. (Special ed.), 24,223

Wu, Y., Luo, A.-L., Li, H.-N., et al. 2011a, Res. Astron. Astrophys., 11, 924

Wu, Y., Singh, H. P., Prugniel, P., Gupta, R., \& Koleva, M. 2011b, A\&A, 525, A71

Yoss, K. M., Neese, C. L., \& Hartkopf, W. I. 1987, AJ, 94, 1600 